\title{
K-SPACE FUNCTION SPACES
}

\author{
R. A. McCOY \\ Department of Mathematics \\ Virginia Polytechnic Institute \\ and State University \\ Blacksburg, Virginia 24061 U.S.A. \\ (Received January 28, 1980)
}

ABSTRACT. A study is made of the properties on $X$ which characterize when $C_{\pi}(X)$ is a k-space, where $C_{\pi}(X)$ is the space of real-valued continuous functions on $X$ having the topology of pointwise convergence. Other properties related to the k-space property are also considered.

KEY WORDS AND PHRASES. Function spaces, k-spaces, Sequential spaces, Fréchet spaces, Countable tightness, k-countable, $\tau$-countable.

1980 MATHEMATICS SUBJECT CLASS IFICATION CODES. Primary, 54C35; secondary, 54D50, 54D55, 54D20.

1. INTRODUCTION.

If $X$ is a topological space, the notation $C(X)$ is used for the space of all real-valued continuous functions on $X$. One of the natural topologies on $C(X)$ is the topology of pointwise convergence, where subbasic open sets are those of the form

$$
\llbracket x, v \rrbracket \equiv\{f \in C(X) \mid f(x) \in V\}
$$


for $\mathrm{x} \in \mathrm{X}$ and $\mathrm{V}$ open in the space of real numbers, $\mathbf{R}$, with the usual topology. The space $C(X)$ with the topology of pointwise convergence will be denoted by $\mathrm{C}_{\pi}(\mathrm{X})$.

For a completely regular space $X, C_{\pi}(X)$ is first countable, in fact metrizable, if and only if $\mathrm{x}$ is countable [2]. The purpose of this paper is to show to what extent this result can be extended to properties more general than first countability, such as that of being a k-space. Throughout this paper all spaces will be assumed to be completely regular $\mathrm{T}_{1}$-spaces.

We first recall the definitions of certain generalizations of first countability. The space $\mathrm{X}$ is a Fréchet space if whenever $\mathrm{x} \in \overline{\mathrm{A}} \subseteq \mathrm{X}$, there exists a sequence in $A$ which converges to $x$. The space $X$ is a sequential space if the open subsets of $X$ are precisely those subsets $U$ such that whenever a sequence converges to an element of $U$, the sequence is eventually in $U$. Also $X$ is a $\underline{k}-$ space if the closed subsets of $\mathrm{X}$ are precisely those subsets $A$ such that for every compact' subspace $K \subseteq X, A \cap K$ is closed in $K$. Finally $X$ has countable tightness if whenever $x \in \bar{A} \subseteq X$, there exists a countable subset $B \subseteq A$ such that $x \in \bar{B}$. The following diagram shows the implications between these properties.

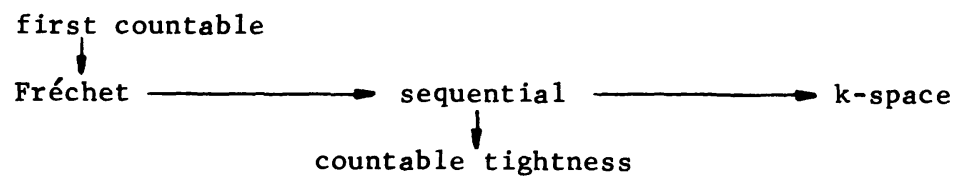

We will show that the Fréchet space, sequential space, and k-space properties are equivalent for $C_{\pi}(X)$. In order to characterize these properties for $c_{\pi}(X)$ in terms of internal properties of $X$, we will need to make some additional definitions. Let $f(X)$ be the set of all nonempty finite subsets of $X$. A collection $u$ of open subsets of $X$ is an open cover for finite subsets of $X$ if for every $A \in \sigma_{n}(X)$, there exists a $U \in U$ such that $A \subseteq U$. If $\left\{U_{n}\right\}$ is a sequence of collections of subsets of $x$, a string from $\left\{u_{n}\right\}$ is a sequence $\left\{U_{n}\right\}$ such that $U_{n} \in u_{n}$ 
for every $n \in \mathbb{N}$ ( $\mathbb{N}$ is the set of natural numbers). In addition, we will say that $\left\{U_{n}\right\}$ is residually covering if for every $x \in X$, there exists an $N \in N$ such that for a $11 \mathrm{n} \geq \mathrm{N}, \mathrm{x} \in \mathrm{U}_{\mathrm{n}}$.

THEOREM 1. The following are equivalent.

(a) $C_{\pi}(X)$ is a Fréchet space.

(b) $C_{\pi}(X)$ is a sequential space.

(c) $c_{\pi}(X)$ is a k-space.

(d) Every sequence of open covers for finite subsets of $X$ has a residually covering string.

PROOF. (d) $\Rightarrow$ (a). Suppose that every sequence of open covers for finite subsets of $X$ has a residually covering string. Let $F$ be a subset of $C_{\pi}(X)$, and let $\mathrm{f}$ be an accumulation point of $\mathrm{F}$ in $\mathrm{C}_{\pi}(\mathrm{X})$. Then for every $\mathrm{n} \in \mathbf{N}$ and $A=$ $\left\{\mathrm{x}_{1}, \ldots, \mathrm{x}_{\mathrm{k}}\right\} \in \mathscr{F}(\mathrm{x})$, we may choose an

$$
\mathrm{f}_{\mathrm{n}, \mathrm{A}} \in \mathrm{F} \cap\left[\mathrm{x}_{1},\left(\mathrm{f}\left(\mathrm{x}_{1}\right)-\frac{1}{\mathrm{n}}, \mathrm{f}\left(\mathrm{x}_{1}\right)+\frac{1}{\mathrm{n}}\right) \rrbracket \cap \ldots \cap \llbracket \mathrm{x}_{\mathrm{k}},\left(\mathrm{f}\left(\mathrm{x}_{\mathrm{k}}\right)-\frac{1}{\mathrm{n}}, \mathrm{f}\left(\mathrm{x}_{\mathrm{k}}\right)+\frac{1}{\mathrm{n}}\right) \rrbracket .\right.
$$

Also define $U(n, A)=\left\{x \in X|| f_{n, A}(x)-f(x) \mid<\frac{1}{n}\right\}$, which is an open subset of $X$. Then for each $n \in \mathbb{N}$, define $u_{n}=\{U(n, A) \mid A \in F(X)\}$, which is an open cover for finite subsets of $X$. Now $\left\{u_{n}\right\}$ has a residually covering $\operatorname{string}\left\{U\left(n, A_{n}\right\}\right.$, so that for every $n \in \mathbb{N}$, we may define $f_{n}=f_{n, A_{n}}$.

We wish to establish that $\left\{f_{n}\right\}$ converges to $f$ in $c_{\pi}(x)$. So let $x \in x$, and let $\epsilon>0$. There is an $N \in \mathbb{N}$ with $N \geq \frac{1}{\epsilon}$ such that for every $n \geq N, x \in U\left(n, A_{n}\right)$. But then if $\mathrm{n} \geq \mathrm{N}$,

$$
\left|f_{n}(x)-f(x)\right|=\left|f_{n, A_{n}}(x)-f(x)\right|<\frac{1}{n} \leq \frac{1}{\mathbb{N}} \leq \epsilon .
$$

Therefore $\left\{f_{n}(x)\right\}$ converges to $f(x)$ for every $x \in X$, so that $\left\{f_{n}\right\}$ converges to $f$ in $C_{\pi}(X)$. Hence $C_{\pi}(X)$ must be a Fréchet space.

$(c) \Rightarrow(d)$. Suppose $X$ has a sequence $\left\{u_{n}\right\}$ of open covers for finite subsets such that no string from $\left\{u_{n}\right\}$ is residually covering. Let $v_{1}=u_{1}$, and for each $n>1$, let ${ }^{\prime} n$ be an open cover for finite subsets of $X$ which refines both $v_{n-1}$ 
and $u_{n}$. For every $n \in \mathbb{N}$ and $A \in \mathscr{F}(X)$, let $U(n, A) \in v_{n}$ such that $A \subseteq U(n, A)$, and let $f_{n, A} \in C(X)$ be such that $f_{n, A}(A)=\left\{\frac{1}{n}\right\}, f_{n, A}(X \backslash U(n, A))=\{n\}$, and $f_{n, A}(X) \subseteq\left[\frac{1}{n}, n\right]$. Then define

$$
F=\left\{f_{n, A} \mid n \in N \text { and } A \in \mathscr{F}(X)\right\},
$$

and also define $F^{*}=\bar{F} \backslash\left\{c_{0}\right\}$ in $C_{\pi}(X)$, where $c_{0}$ is the constant zero function.

First we establish that $F^{*}$ is not closed in $C_{\pi}(X)$ by showing that $c_{0}$ is an accumulation point of $F$ in $C_{\pi}(X)$. To do this, let $w=\llbracket\left[x_{1}, v_{1} \rrbracket \cap \ldots \cap \llbracket x_{k}, v_{k} \rrbracket\right.$ be an arbitrary basic neighborhood of $c_{0}$ in $c_{\pi}(x)$. If $A=\left\{x_{1}, \ldots, x_{k}\right\}$ and $n \in \mathbb{N}$ such that $\frac{1}{n} \in v_{1} \cap \ldots \cap v_{k}$, then $f_{n, A} \in W \cap F$.

We will then obtain that $C_{\pi}(X)$ is not a k-space, as desired, if we can show that the intersection of $F^{*}$ with each compact subspace of $C_{\pi}(X)$ is closed in that compact subspace. To this end, let $\mathrm{K}$ be an arbitrary compact subspace of $C_{\pi}(X)$. Then for every $x \in X$, the orbit $\{f(x) \mid f \in K\}$ is bounded in $R$. For every $x \in X$, define $M(x)=\sup \{f(x) \mid f \in K\}$, and also for every $m \in \mathbb{N}$, define $x_{m}=$ $\{x \in X \mid M(x) \leq m\}$. Note that $x=U\left\{x_{m} \mid m \in \mathbb{N}\right\}$, and that for every $m, x_{m} \leq x_{m+1}$. Suppose, by way of contradiction, that for every $m, n \in \mathbf{N}$, there exists a $\mathrm{k} \geq \mathrm{n}$ and $\mathrm{v} \in \mathrm{v}_{\mathrm{k}}$ such that $\mathrm{x}_{\mathrm{m}} \subseteq \mathrm{v}$. We define, by induction, a string $\left\{\mathrm{U}_{\mathrm{n}}\right\}$ from $\left\{u_{n}\right\}$. First there exists a $k_{1} \geq 1$ and $v_{1} \in v_{k_{1}}$ such that $x_{1} \subseteq v_{1}$. For each $i=1, \ldots, k_{1}$, choose $u_{i} \in u_{i}$ so that $v_{1} \subseteq u_{i}$. Now suppose $k_{m}$ and $U_{1}, \ldots, u_{m}$ have been defined. Then there exists a $k_{m+1} \geq k_{m}+1$ and $v_{m+1} \in v_{k_{m+1}}$ such that $\mathrm{x}_{\mathrm{m}+1} \subseteq \mathrm{v}_{\mathrm{m}+1}$. For each $\mathrm{i}=\mathrm{k}_{\mathrm{m}}+1, \ldots, \mathrm{k}_{\mathrm{m}+1}$, choose $\mathrm{U}_{i} \in u_{i}$ so that $\mathrm{v}_{\mathrm{m}+1} \subseteq \mathrm{U}_{i}$. This defines string $\left\{U_{n}\right\}$, which we know to not be residually covering. Let $x \in \mathrm{x}$ be arbitrary. There is an $\mathrm{m} \in \mathbf{N}$ such that $\mathrm{x} \in \mathrm{x}_{\mathrm{m}}$. Let $\mathrm{n} \geq \mathrm{k}_{\mathrm{m}}$. There is a $\mathrm{j} \geq \mathrm{m}$ such that $\mathrm{k}_{\mathrm{j}-1}+1 \leq \mathrm{n} \leq \mathrm{k}_{\mathrm{j}}$. Then $\mathrm{x} \in \mathrm{x}_{\mathrm{m}} \subseteq \mathrm{x}_{\mathrm{j}} \subseteq \mathrm{v}_{\mathrm{j}} \subseteq \mathrm{U}_{\mathrm{n}} \cdot$ But this says that $\left\{U_{n}\right\}$ is residually covering, which is a contradiction.

We have just established that there exist $m, n \in \mathbf{N}$ such that for every $k \geq n$ and for every $v \in v_{k}, x_{m} \notin v$. Then define $M=\max \{m, n\}$, let $x_{0} \in x$ be 
arbitrary, and define $W=\left[x_{0},\left(-\frac{1}{M}, \frac{1}{M}\right)\right]$, which is a neighborhood of $c_{0}$ in $C_{\pi}(X)$. Suppose $f \in W \cap F$. Then there exists a $k \in \mathbf{N}$ and $A \in F(X)$ such that $\mathrm{f}=\mathrm{f}_{\mathrm{k}, \mathrm{A}^{\cdot}}$. Since $\frac{1}{\mathrm{k}} \leq \mathrm{f}\left(\mathrm{x}_{\mathrm{o}}\right)<\frac{1}{\mathrm{M}}$, then $\mathrm{k}>\mathrm{M} \geq \mathrm{n}$. Thus $\mathrm{x}_{\mathrm{m}} \notin \mathrm{U}(\mathrm{k}, \mathrm{A})$, so that there exists an $x_{1} \in x_{m} \backslash U(k, A)$. But then $f\left(x_{1}\right)=k>M \geq m \geq M\left(x_{1}\right)$, so that $\notin K$. Therefore $\mathrm{W} \cap \mathrm{F} \cap \mathrm{K}=\varnothing$, so that $c_{0}$ is not an accumulation point of $F * \cap K$ in $K$. Hence $F^{*} \cap \mathrm{K}$ must be closed in $K$. Since $K$ was arbitrary, we obtain that $C_{\pi}(X)$ is not a $\mathrm{k}$-space.

THEOREM 2. $C_{\pi}(X)$ has countable tightness if and only if every open cover for finite subsets of $X$ has a countable subcover for finite subsets of $X$.

PROOF. Suppose that every open cover for finite subsets of $X$ has a countable subcover for finite subsets of $X$. Let $F$ be a subset of $C_{\pi}(X)$, and let $f$ be an accumulation point of $F$ in $C_{\pi}(X)$. Then for each $n \in \mathbb{N}$ and $A=\left\{x_{1}, \ldots, x_{k}\right\}$ $\epsilon \mathscr{F}(\mathrm{X})$, choose

$$
f_{n, A} \in F \cap \mathbb{I} x_{1},\left(f\left(x_{1}\right)-\frac{1}{n}, f\left(x_{1}\right)+\frac{1}{n}\right) \rrbracket \cap \ldots \cap\left[x_{k},\left(f\left(x_{k}\right)-\frac{1}{n}, f\left(x_{k}\right)+\frac{1}{n}\right) \rrbracket .\right.
$$

Also let $U(n, A)=\left\{x \in X|| f_{n, A}(x)-f(x) \mid<\frac{1}{n}\right\}$, which is an open subset of $x$. Then for each $n \in \mathbb{N},\{U(n, A) \mid A \in \mathscr{F}(X)\}$ is an open cover for finite subsets of $X$. So for each $n \in \mathbb{N}$, there exists a sequence $\{A(n, i) \mid i \in \mathbb{N}\}$ from $\mathscr{F}(X)$ such that $\{U(n, A(n, i)) \mid i \in \mathbb{N}\}$ is a cover for finite subsets of $X$. Then define $G=$ $\left\{\mathrm{f}_{\mathrm{n}, \mathrm{A}(\mathrm{n}, \mathrm{i})} \mid \mathrm{n}, \mathrm{i} \in \mathbf{N}\right\}$.

To see that $f \in \bar{G}$, let $W=\left[x_{1}, v_{1}\right] \cap \ldots \cap\left[\left[x_{k}, v_{k} \rrbracket\right.\right.$ be a neighborhood of $f$ in $C_{\pi}(X)$. Let $A=\left\{x_{1}, \ldots, x_{k}\right\}$, and choose $n \in \mathbb{N}$ so that $\left(f\left(x_{j}\right)-\frac{1}{n}\right.$, $\left.f\left(x_{j}\right)+\frac{1}{n}\right) \subseteq v_{j}$ for each $j=i, \ldots, k$. Then there is an $i \in \mathbb{N}$ such that $A \subseteq U(n, A(n, i))$. So for each $x \in A,\left|f_{n, A(n, i)}(x)-f(x)\right|<\frac{1}{n}$, and hence $\mathrm{f}_{\mathrm{n}, \mathrm{A}(\mathrm{n}, \mathrm{i})} \in \mathrm{W}$.

Conversely, suppose that $C_{\pi}(X)$ has countable tightness, and let $u$ be an open cover for finite subsets of $X$. For each $A \in F(X)$, let $U(A) \in U$ be such that $A \subseteq U(A)$. Also for each $n \in \mathbb{N}$ and $A \in \mathscr{F}(X)$, let $f_{n, A} \in C(X)$ be such that 
$f_{n, A}(A)=\left\{\frac{1}{n}\right\}, f_{n, A}(X \backslash U(A))=\{n\}$, and $f_{n, A}(X) \subseteq\left[\frac{1}{n}, n\right]$. Then define $F=$ $\left\{f_{n, A} \mid n \in N\right.$ and $\left.A \in \mathscr{F}(X)\right\}$.

Since the constant zero function, $c_{0}$, is an accumulation point of $F$, then there is a countable subset $G$ of $F$ such that $c_{0} \in \bar{G}$. There are sequences $\left\{n_{i}\right\} \subseteq \mathbb{N}$ and $\left\{A_{i}\right\} \subseteq \mathscr{F}(X)$ so that $G=\left\{f_{n_{i}, A_{i}} \mid i \in \mathbb{N}\right\}$.

To see that $\left\{U\left(A_{i}\right) \mid i \in N\right\}$ is a cover for finite subsets of $X$, let $A=$ $\left\{x_{i}, \ldots, x_{k}\right\} \in F(x)$. Then there exists an $i \in \mathbb{N}$ such that $f_{n_{i}, A_{i}} \in \mathbb{I} x_{i}$, $(-1,1) \rrbracket \cap \ldots \cap\left[x_{k},(-1,1) \rrbracket\right.$. But this means that $A \subseteq U\left(A_{i}\right)$, so that $\left\{U\left(A_{i}\right) \mid i \in \mathbb{N}\right\}$ is indeed a cover for finite subsets of $x$.

Let us now give names to the two properties of $\mathrm{X}$ which are expressed in Theorems 1 and 2 . We will call X k-countable whenever $C_{\pi}(X)$ is a k-space, and we will call X $\underline{\tau \text {-countable }}$ whenever $C_{\pi}(X)$ has countable tightness. We state some immediate facts about these properties.

PROPOSITION 3. Every countable space is k-countable.

PROPOSITION 4. Every k-countable space is T-countable.

PROPOSITION 5. Every $\tau$-countable space is Lindelöf.

PROOF. Let $X$ be $\tau$-countable, and let $u$ be an open cover of $X$. Let $v$ be the family of all finite unions of members of $u$. Then $v$ is an open cover for finite subsets of $X$, so that it has a countable subcover $W$ for finite subsets of $X$. Each member of $u_{1}$ is a finite union of members of $u$, so that since in covers $x$, then $u$ has a countable subcover. $\square$

This means that if $C_{\pi}(X)$ has countable tightness, $X$ must be Lindelöf. In particular, $C_{\pi}\left(\Omega_{0}\right)$ does not have countable tightness, where $\Omega_{0}$ is the space of countable ordinals with the order topology. This is in contrast to $C_{\pi}(\Omega)$, which we see from the next proposition has countable tightness, where $\Omega=\Omega_{0} \|\left\{\omega_{1}\right\}$. PROPOS ITION 6. If $x^{n}$ is Lindelöf for every $n \in \mathbb{N}$, then $X$ is $\tau$-countable. PROOF. Let $x^{n}$ be Lindelöf for every $n \in \mathbb{N}$, and let $u$ be an open cover for finite subsets of $x$. For each $n \in \mathbb{N}$, let $u_{n}=\left\{U^{n} \subseteq x^{n} \mid U \in u\right\}$. Since $u$ is an 
open cover for finite subsets of $x$, then each $u_{n}$ is an open cover of $x^{n}$. So for each $n \in \mathbb{N}, u$ has a countable subcollection $v_{n}$ such that $\left\{U^{n} \mid U \in v_{n}\right\}$ covers $x^{n}$. But then $U\left\{v_{n} \mid n \in \mathbb{N}\right\}$ is a countable subcollection of $u$ which is a cover for finite subsets of $x$.

COROLLARY 7. Every compact space is T-countable, and every separable metric space is r-countable.

We now examine some properties of k-countable spaces.

PROPOSITION 8. Every closed subspace of a k-countable space is k-countable.

PROOF. Let $X$ be a k-countable space, and let $Y$ be a closed subspace of $X$. Let $\left\{v_{n}\right\}$ be a sequence of open covers for finite subsets of $Y$. For each $n \in \mathbf{N}$, let $u_{n}=\left\{V \cup(X \backslash Y) \mid V \in v_{n}\right\}$, which is an open cover for finite subsets of $X$. Now $\left\{u_{n}\right\}$ has a residually covering string $\left\{V_{n} \cup(X \backslash Y)\right\}$, where each $v_{n} \in v_{n}$. But then $\left\{v_{n}\right\}$ is a residually covering string from $\left\{v_{n}\right\}$.

PROPOSITION 9. Every continuous image of a k-countable space is k-countable.

PROOF. Let $X$ be $k$-countable, and let $\mathrm{f}: \mathrm{X} \rightarrow \mathrm{Y}$ be a continuous surjection. Let $\left\{y_{n}\right\}$ be a sequence of open covers for finite subsets of $Y$. For each $n \in \mathbb{N}$, let $u_{n}=\left\{f^{-1}(v) \mid v \in v_{n}\right\}$, which is an open cover for finite subsets of $x$. Now $\left\{u_{n}\right\}$ has a residually covering string $\left\{f^{-1}\left(v_{n}\right)\right\}$, where each $v_{n} \in v_{n}$. But then $\left\{\mathrm{v}_{\mathrm{n}}\right\}$ is a residually covering string from $\left\{\mathrm{v}_{\mathrm{n}}\right\}$.

In the next proposition, we use the term covering string, by which we mean a string which is itself a cover of the space.

PROPOSITION 10. If $\mathrm{X}$ is k-countable, then every sequence of open covers of $\mathrm{X}$ has a covering string.

PROOF. Let $\left\{u_{n}\right\}$ be a sequence of open covers of $x$. For each $n \in N$, let

$$
v_{n}=\left\{U_{n} U \cdots \| U_{n+k+1} \mid k \in \mathbb{N} \text { and each } U_{i} \in u_{i}\right\} \text {, }
$$

which is an open cover for finite subsets of $x$. Thus $\left\{V_{n}\right\}$ has a residually covering string $\left\{\mathrm{v}_{\mathrm{n}}\right\}$. Now $\mathrm{v}_{1}=\mathrm{u}_{1} \mathrm{U} \cdot \cdots \mathrm{u}_{\mathrm{k}_{1}}$ for some $\mathrm{k}_{1} \in \mathbb{N}$. Also $\mathrm{v}_{\mathrm{k}_{1}+1}=$ 
$\mathrm{U}_{\mathrm{k}_{1}+1} U \cdots \cup \mathrm{U}_{\mathrm{k}_{2}}$ for some $\mathrm{k}_{2} \in \mathrm{N}$ with $\mathrm{k}_{2}>\mathrm{k}_{1}$. Continuing by induction, we can define an increasing sequence $\left\{k_{i}\right\}$ such that each $v_{k_{i}+1}=U_{k_{i}+1} U \cdots \cup U_{k_{i+1}}$. This defines $U_{n}$ for each $n \in \mathbb{N}$. To see that $\left\{U_{n}\right\}$ is a covering string from $\left\{u_{n}\right\}$ let $x \in X$. Then there exists an $N \in N$ such that for all $n \geq N, x \in v_{n}$. Since $\left\{k_{i}\right\}$ is increasing, there is some $i$ such that $k_{i} \geq N$. Then $x \in v_{k_{i}+1}=$ $\mathrm{U}_{\mathrm{k}_{\mathrm{i}}+1} U \cdots \mathrm{U}_{\mathrm{k}_{\mathrm{i}+1}}$, so that $\mathrm{x}$ is indeed in some $\mathrm{U}_{\mathrm{n}}$.

We next give an important example of a space which is not k-countable.

EXAMPLE 11. The closed unit interval, $I$, is not k-countable.

PROOF. For each $n \in \mathbb{N}$, let $u_{n}$ be the set of all open intervals in $I$ having diameter less than $\frac{1}{2^{n}}$. Suppose $\left\{u_{n}\right\}$ were to have a covering string $\left\{U_{n}\right\}$. Then since $I$ is connected, there would be a simple chain $\left\{U_{n_{1}}, \ldots, U_{n_{k}}\right\}$ from 0 to 1 . That is, $0 \in U_{n_{1}}, 1 \in U_{n_{k}}$, and for each $1 \leq i \leq k-1$, there is a $t_{i} \in U_{n_{i}} \cap U_{n_{i+1}}$. But then

$$
\begin{aligned}
1 & \leq\left|1-t_{k-1}\right|+\left|t_{k-1}-t_{k-2}\right|+\ldots+\left|t_{2}-t_{1}\right|+\left|t_{1}\right| \\
& <\frac{1}{2^{n} k}+\frac{1}{2^{n} k-1}+\ldots+\frac{1}{2^{n_{2}}}+\frac{1}{2^{n} 1} \\
& \leq \frac{1}{2}+\frac{1}{2^{2}}+\cdots+\frac{1}{2^{k}}<1 .
\end{aligned}
$$

This is a contradiction, so that $\left\{u_{n}\right\}$ cannot have a covering string. Therefore, by Proposition 10, I is not k-countable.

The next three results are consequences of Example 11 .

EXAMPLE 12. The Cantor set, $\mathbf{K}$, is not k-countable.

PROOF. Since there exists a continuous function from $\mathbb{K}$ onto $I$, then $\mathbf{K}$ cannot be k-countable because of Proposition 9 and Example 11.

Our next proposition then follows from Example 12 and Proposition 8.

PROPOSITION 13. No k-countable space contains a Cantor set.

PROPOSITION 14. Every k-countable space is o-dimensional. 
PROOF. Let $\mathrm{X}$ be $\mathrm{k}$-countable, let $\mathrm{x} \in \mathrm{X}$, and let $\mathrm{U}$ be an open neighborhood of $x$ in $X$. Since $X$ is completely regular, there exists an $f \in C(X)$ such that $f(x)=0, f(X \backslash U)=\{1\}$, and $f(X) \subseteq I$. Since I is not k-countable by Example 11, and since $f(X)$ is $k$-countable by Proposition 9, then there exists a $t \in I \backslash f(X)$. Thus $[0, t) \cap f(X)$ is both open and closed in $f(X)$, so that $f^{-1}([0, t)$ ) is an open and closed neighborhood of $x$ contained in $U$.

With all these necessary conditions which k-countable spaces must satisfy, one might wonder whether there exists an uncountable k-countable space. This is answered by the next two examples.

We will call a space $X$ virtually countable if there exists a finite subset $F$ of $X$ such that for every open subset $U$ of $X$ with $F \subseteq U$, it is true that $X \backslash U$ is countable. Notice that a first countable virtually countable space is countable.

PROPOSITION 15. Every virtually countable space is k-countable.

PROOF. Let $F$ be a finite subset of $X$ such that every open $U$ in $X$ with $F \subseteq U$ has countable complement, and let $\left\{u_{n}\right\}$ be a sequence of open covers for finite subsets of $X$. First let $U_{1} \in u_{1}$ be such that $F \subseteq U_{1}$. Then $X \backslash U_{1}$ is countable; say $x \backslash U_{1}=\left\{x_{11}, x_{12}, x_{13}, \ldots\right\}$. Let $U_{2} \in u_{2}$ be such that $F \cup\left\{x_{11}\right\} \subseteq U_{2}$. Now $X \backslash U_{2}$ is also countable; say $X \backslash U_{2}=\left\{x_{21}, x_{22}, x_{23}, \ldots\right.$. Let $U_{3} \in u_{3}$ be such that $F \cup\left\{x_{11}, x_{12}, x_{21}\right\} \subseteq U_{3}$. Continuing by induction, we may define string $\left\{U_{n}\right\}$ from $\left\{u_{n}\right\}$ such that for each $n, U_{n}=X \backslash\left\{x_{n 1}, x x_{n 2}\right.$, $\left.x_{n 3}, \cdots\right\}$ and

$$
F \|\left\{x_{11}, \ldots, x_{1 n}, x_{21}, \ldots, x_{2, n-1}, \ldots, x_{n 1}\right\} \subseteq U_{n+1} \cdot
$$

To see that every elęment of $x$ is residually in $\left\{U_{n}\right\}$, let $x \in X$. If $x \in \bigcap_{n=1}^{\infty} U_{n}$, then $x$ is residually in $\left\{U_{n}\right\}$. If $x \in \bigcap_{n=1}^{\infty} U_{n}$, then let $i$ be the first integer such that $x \notin U_{i}$. Then $x=x_{i j}$ for some $j$, so that for every $n \geq i+j, x \in U_{n}$. Therefore $\mathrm{x}$ is residually in $\left\{\mathrm{U}_{\mathrm{n}}\right\}$. 
EXAMPLE 16. The space of ordinals, $\Omega$, which are less than or equal to the first uncountable ordinal is k-countable.

PROOF. It is easy to see that $\Omega$ is virtually countable.

EXAMPLE 17. The Fortissimo space, $\mathbb{F}$, is $k$-countable, where $\mathbb{F}$ is $\mathbf{R}$ with the following topology: each $\{t\}$ is open for $t \neq 0$, and the open sets containing 0 are the sets containing 0 which have countable complements. Also $\mathbb{F}^{2}$ is not Lindelöf, which shows that the converse of Proposition 6 is not true.

PROOF. Obviously $\mathbb{F}$ is virtually countable. However, an alternate proof can be obtained from known properties of this space. In particular, it follows from [1] that $C_{\pi}(\mathbb{F})$ is homeomorphic to a $\Sigma$-product of copies of $R$, and from [3] that a $\Sigma$-product of first countable spaces is a Fréchet space.

The spaces in the previous two examples are not first countable. This raises the following question.

QUESTION 18. Is every first countable k-countable space countable?

One well studied example of an uncountable first countable space which is also a o-dimensional Lindelöf space and which does not contain a Cantor set is the Sorgenfrey line. However, in our last example we show that this space is not k-countable, and in fact is not even $\tau$-countable.

EXAMPLE 19. The Sorgenfrey line, $S$, is not $\tau$-countable. This shows that the converse of Proposition 5 is not true.

PROOF. For each $A \in \mathscr{F}(S)$, let $\delta(A)=\frac{1}{2} \min \left\{\left|a-a^{\prime}\right| \mid a, a^{\prime} \in A\right.$, with $\left.a \neq a^{\prime}\right\}$, and let $U(A)=U\{[a, a+\delta(A)) \mid a \in A\}$. Then define $U=\{U(\hat{A}) \mid A \in \mathcal{F}(S)\}$, where $\hat{A}=$ $A \|\{-a \mid a \in A\}$. Clearly $u$ is an open cover for finite subsets of $S$. Then $\left\{U^{2} \mid U \in U\right\}$ is an open cover of $s^{2}$. But each $U^{2}$, for $U \in U$, intersects the set $\left\{(x, y) \in s^{2} \mid x+y=0\right\}$ on a finite set, so that $\left\{U^{2} \mid U \in U\right\}$ has no countable subcover of $s^{2}$. Therefore no countable subcollection of $u$ can cover all doubleton subsets of $\mathrm{S}$. 


\section{REFERENCES}

1. H. H. Corson, Normality in subsets of product spaces, Amer. J. Math. 81 (1959), 785-796.

2. R. A. McCoy, Countability properties of function spaces, to appear in Rocky Mountain J. Math.

3. N. Noble, The continuity of functions on Cartesian products, Trans. Amer. Math. Soc. 149 (1970), 187-198. 


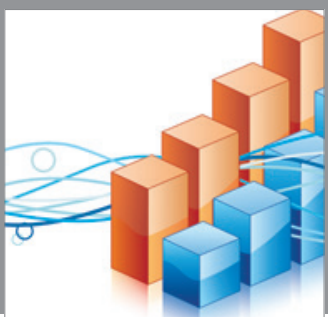

Advances in

Operations Research

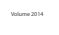

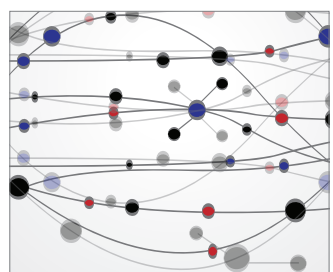

\section{The Scientific} World Journal
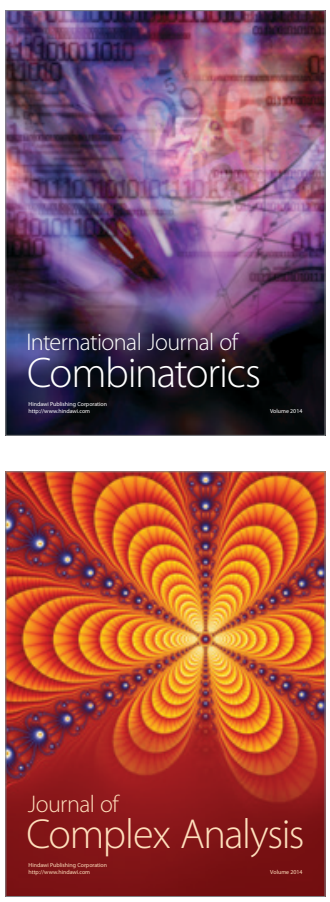

International Journal of

Mathematics and

Mathematical

Sciences
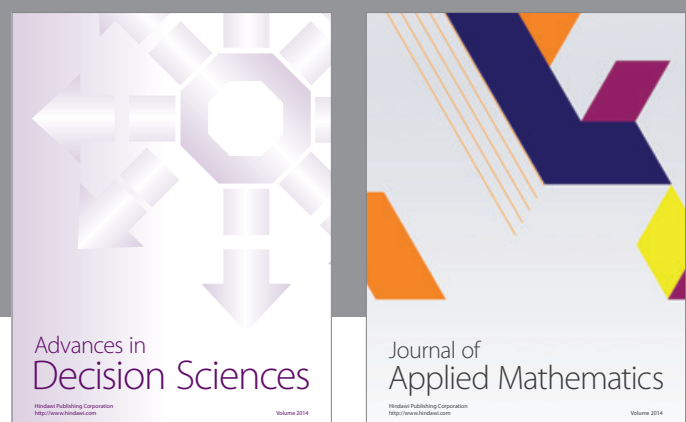

Journal of

Applied Mathematics
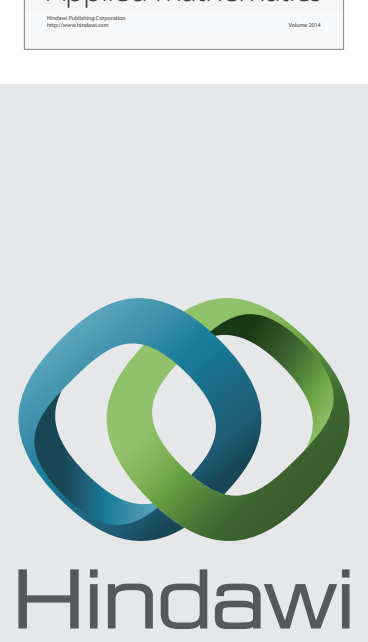

Submit your manuscripts at http://www.hindawi.com
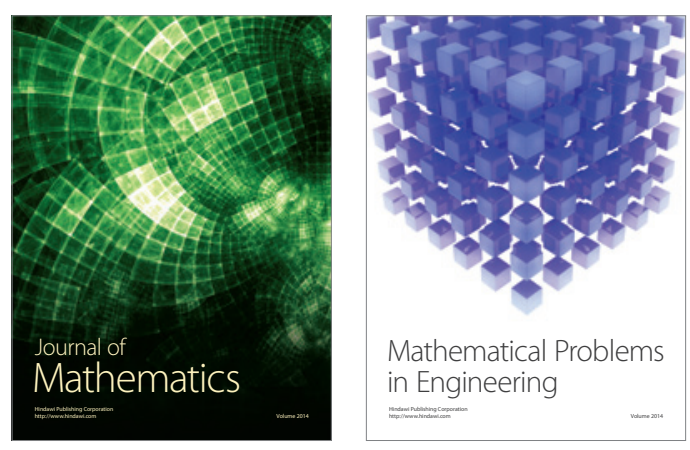

Mathematical Problems in Engineering
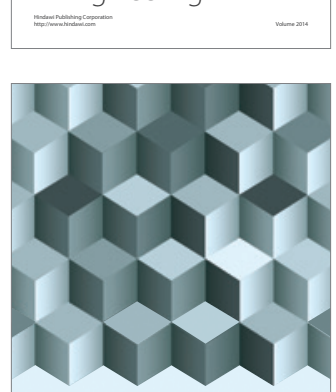

Journal of

Function Spaces
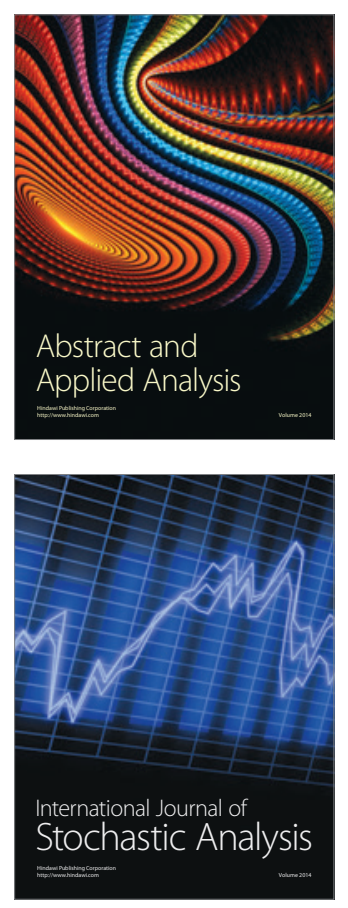

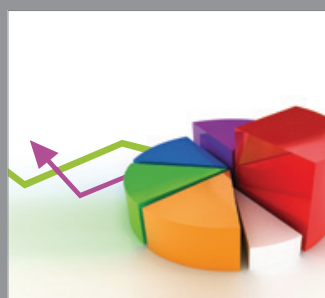

ournal of

Probability and Statistics

Promensencen
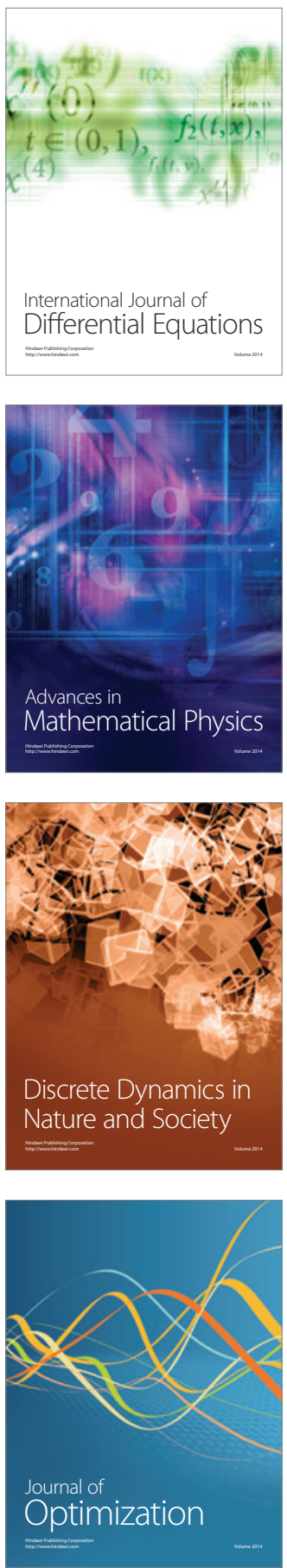